\title{
Effect of Acupuncture on Pregnancy-Related Insomnia and Melatonin: A Single-Blinded, Randomized, Placebo-Controlled Trial
}

This article was published in the following Dove Press journal: Nature and Science of Sleep

\section{Saeedeh Foroughinia (iD) \\ Kamran Hessami ${ }^{2,3}$ \\ Nasrin Asadi ${ }^{3}$ \\ Leila Foroughinia ${ }^{4}$ \\ Mohammadjavad Hadianfard ${ }^{5}$ \\ Azadeh Hajihosseini ${ }^{5}$ \\ Najmeh Pirasteh ${ }^{5}$ \\ Mehrdad Vossoughi (iD ${ }^{6}$ \\ Homeira Vafaei (iD ${ }^{3}$ \\ Azam Faraji ${ }^{3}$ \\ Maryam Kasraeian ${ }^{3}$ \\ Mehrnoosh Doroudchi ${ }^{7}$ \\ Mohammad Rafiee Monjezi iD ${ }^{8}$ \\ Shohreh Roozmeh (iD ${ }^{3}$ \\ Khadije Bazrafshan ${ }^{3}$ \\ 'Obstetrics and Gynecology Department, Shiraz University of Medical Sciences, Shiraz, Iran; ${ }^{2}$ Student Research Committee, Shiraz University of Medical Sciences, Shiraz, Iran; ${ }^{3}$ Maternal-Fetal Medicine Research Center, Shiraz University of Medical Sciences, Shiraz, Iran; ${ }^{4}$ Box Hill Hospital, FRANZCOG, Eastern Health, Victoria, Australia; ${ }^{5}$ Physical Medicine and Rehabilitation Department, Shiraz University of Medical Sciences, Shiraz, Iran; ${ }^{6}$ Oral and Dental Disease Research Center, Department of Dental Public Health, School of Dentistry, Shiraz University of Medical Sciences, Shiraz, Iran; ${ }^{7}$ Department of Immunology, School of Medicine, Shiraz University of Medical Sciences, Shiraz, Iran; ${ }^{8}$ Shiraz Institute for Cancer Research, Shiraz University of Medical Sciences, Shiraz, Iran}

Correspondence: Nasrin Asadi Maternal-Fetal Medicine Research Center, Shiraz University of Medical Sciences, Shiraz, Iran

Tel +98-7| -36128257

Fax +98-71-362128257

Email nasadi2012@yahoo.ca
Objective: The aim of the current study is to evaluate the efficacy and safety of acupuncture on sleep quality and overnight melatonin secretion, measured as urinary 6-sulfatoxymelatonin, in pregnant women.

Patients and Methods: This randomized, parallel, single-blinded (participant), controlled trial was conducted on 72 pregnant women with insomnia. Study participants were randomly assigned to either the intervention, 10 sessions of acupuncture treatment over a 3-week period, or control group by block randomization (1:1). Patients in both groups were evaluated at baseline and post-treatment (third week) using the Pittsburgh Sleep Quality Index (PSQI) score (as the primary outcome) and urinary 6-sulfatoxymelatonin.

Results: Fifty-five of 72 participants completed the study. There was no statistically significant difference regarding PSQI score and 6-sulfatoxymelatonin level between intervention and control groups at the baseline $(P=0.169$ and $P=0.496)$. At the end of the study period, treatment with acupuncture significantly improved the PSQI score $(P<0.001)$ with a large effect size of 3.7 , as well as 6-sulfatoxymelatonin level $(P=0.020)$ with a medium effect size of 0.6 as compared to the control group. No adverse effects were noted during acupuncture sessions and follow-up visits.

Conclusion: Acupuncture was shown to significantly improve the sleep quality in pregnant women, possibly through increasing melatonin secretion, and could be recommended as a low-cost and low-risk alternative treatment to pharmacological therapies.

Keywords: acupuncture, pregnancy, insomnia, melatonin

\section{Introduction}

Insomnia is highly prevalent amongst pregnant women and affects their quality-oflife, but its significance is often underestimated in clinical practice. A recent metaanalysis on sleep quality among pregnant women suggested that an average of $45.7 \%$ pregnant women suffer from poor quality of sleep. ${ }^{1}$ Reports suggest that sleep duration decreases with gestational age during the pregnancy, reaching minimum values in the third trimester. ${ }^{1,2}$ Insomnia during pregnancy appears to have different risk factors in comparison to the non-pregnant state, for example gastroesophageal reflux, increased size of the uterus and anatomical disturbance as well as hormonal change leading to insomnia.,

It has been demonstrated that reduced sleep duration in pregnancy is associated with increased risk of preterm delivery and postnatal depression. ${ }^{4}$ Currently, there is a paucity of knowledge on the exact effects of maternal insomnia on fetal 
development; however, a recent review article suggest that sleep disruption during pregnancy may negatively affect fetal growth and gestational length. ${ }^{5}$ Furthermore, in animal studies, it has been shown that maternal sleep deprivation negatively affects neurogenesis in the fetal brain, resulting in subsequent cognitive impairments. ${ }^{6,7}$ Therefore, prompt treatment of insomnia in pregnant women seems vital to improve both maternal and fetal/ neonatal outcomes.

In the current clinical practice, benzodiazepines and non-benzodiazepine hypnotics are among the most commonly used medications to improve sleep quality in pregnant women. ${ }^{8,9}$ Although no direct teratogenicity has been found in women using benzodiazepines or zolpidem, ${ }^{8}$ it is recommended to avoid routinely prescribing these medications due to higher incidence of adverse perinatal outcomes such as preterm delivery, ${ }^{10}$ low birth weight, ${ }^{10}$ cesarean delivery, ${ }^{11}$ and neonatal abstinence syndrome (especially with benzodiazepines). ${ }^{12}$

Acupuncture is among the popular alternative therapeutic modalities for treatment of insomnia. ${ }^{13,15}$ Interestingly, a recent survey in the United States showed that insomnia is one of the top 10 indications among patients visiting an acupuncture clinic. ${ }^{16}$ In a systematic review on the efficacy of acupuncture for insomnia in a non-pregnant population, acupuncture seems to be a safe and effective method for improving sleep quality. ${ }^{13}$ However, due to discrepancies in the quality of studies, it is premature to make any firm conclusions.

Recent reports suggest that acupuncture can increase the serum concentration of melatonin. ${ }^{17,18}$ Since decreased levels of melatonin are associated with sleep disturbances during pregnancy, ${ }^{19}$ acupuncture may be one promising candidate for relieving pregnancy-related insomnia by upregulating melatonin level. To our knowledge, there has been only one study assessing the effectiveness of acupuncture for treating pregnancy-related insomnia. ${ }^{14}$ The mentioned study was performed on 12 pregnant women in the acupuncture group and 10 in the control group, receiving only sleep hygiene education (gestational age ranging from 15-30 weeks) for 8-12 acupuncture sessions during an 8 -week period. The results suggested that acupuncture may improve sleep quality during pregnancy; however, it was limited by methodological inadequacy and the small number of participants.

We designed a randomized controlled trial to investigate the efficacy and safety of acupuncture on sleep quality and urine 6-sulfatoxymelatonin, for the first time, among pregnant women suffering from insomnia.

\section{Patients and Methods}

\section{Trial Design and Study Subjects}

The current study was a single-blinded (participant), parallel, randomized controlled trial conducted on 72 pregnant women with insomnia referring to Motahari and Hafez clinics, affiliated to Shiraz University of Medical Sciences, from December 2017 to August 2018. The study was conducted in accordance with the Declaration of Helsinki and the protocol was approved by the Ethics Committee of Shiraz University of Medical Sciences (IR.SUMS.MED. REC.1396.77) and all participants signed the written informed consent before participation in the trial. This trial was registered at Iranian Registry of Clinical Trials as IRCT20140525017827N3. This study was in accordance with the Consolidated Standards of Reporting Trials (CONSORT) and Standards for Reporting Interventions in Clinical Trials of Acupuncture (STRICTA) guidelines for designing and reporting controlled trials. ${ }^{20,21}$

\section{Inclusion/Exclusion Criteria}

Inclusion criteria for the study were the following: 1) maternal age $20-45$ years; 2 ) being in second or third trimester of pregnancy, but has at least 4 weeks left to delivery date; 3 ) documentation of short-term insomnia according to International Classification of Sleep Disorders, Third Edition (ICSD-3); ${ }^{22}$ 4) baseline Pittsburgh Sleep Quality Index (PSQI) score more than five; and 5) having night sleep disturbance for at least 4 weeks prior to study, but not before pregnancy. Exclusion criteria were the following: 1) any chronic medical illnesses; 2) history of any psychiatric disorders; 3) history of any sleep disturbance before pregnancy; 4) use of hypnotic drugs; 5) history of alcohol or drug abuse; 6) bleeding tendency and signs of infection at the acupoints; 7) sleep apnea; and 8) high risk complicated pregnancies such as multiple gestation, gestational diabetes mellitus, preeclampsia, fetal growth restriction, etc. Additionally, pregnant women with a night-shift job (ie, nurses) were excluded from the study because of the altered melatonin secretion pattern due to light exposure at night. $^{23}$

\section{Randomization, Allocation, and Blinding}

Block randomization was done using a computergenerated random number list, with a block size of four 
(random list generated with SPSS 17.0 (SPSS Inc., Chicago, IL)), provided by an investigator with no clinical involvement in the trial. The sealed envelope containing an allocation sequence number for each patient will be regarded as a means of allocation concealment.

The outcome assessor, statistician, and study subjects were blinded throughout the study period. This means that those participants who had a previous history of acupuncture treatment and acknowledged the effects of acupoints were excluded. The acupuncturist used a neutral approach when communicating with participants and avoided revealing cues that might indicate whether acupuncture with real or sham needles are being done. However, the acupuncturist was not blinded due to the nature of the intervention. This method of blinding has been used in previous acupuncture studies and shown to be a highquality method for blinding participants. ${ }^{24,25}$

\section{Interventions}

In the intervention group, standard acupuncture was performed for 10 sessions during a 3 -week period (4 times in the first week and 3 times per week for the following weeks). Sterilized stainless-steel needles $(0.3 * 40$ and $0.3^{*} 25 \mathrm{~mm}$ ) were inserted, forming a 90-degree angle with the skin surface and rotated anti-clockwise. The acupoints used in this study include KI-3 (Taixi), HT-7 (Shenmen), ST-36 (Zusanli), PC-6 (Neiguan), DU-20 (Baihui), GB-20 (Fengchi), and EX-8 (Anmian 1). The details on the anatomical location of these points are reported in Table 1.

The acupoints were obtained from an in-depth review of literature for finding acupoints used for relieving insomnia, which are considered safe during pregnancy. ${ }^{13,26,27}$ The expert acupuncture practitioner with $>15$ years of experience performed needle manipulation (manual) until the study subject achieved proper needling sensation "De Qi", then the needles were left in place for approximately 30 minutes.

In the control group, only sleep hygiene education and fake acupuncture with blunt and non-penetrating needles were performed by superficial contact of needles in the same acupoints as real acupuncture. Thus, the aim was not to perform manual manipulation or achieving "De Qi" sensation. This placebo method has been used in previous studies of acupuncture and shown to be a valid technique for blinding of participants in the control group. ${ }^{24}$ In both groups, sleep hygiene education was given to each participant by one of the investigators based on "sleep
Table I Description of Acupoints Used in the Current Study

\begin{tabular}{|l|l|}
\hline $\begin{array}{l}\text { Needling } \\
\text { Point }\end{array}$ & Location \\
\hline KI-3 (Taixi) & $\begin{array}{l}\text { Midway between the medial malleolus and medial } \\
\text { border of the achilles tendon }\end{array}$ \\
\hline $\begin{array}{l}\text { HT-7 } \\
\text { (Shenmen) }\end{array}$ & $\begin{array}{l}\text { On the radial side of the tendon of flexor carpi ulnaris } \\
\text { muscle at the wrist crease }\end{array}$ \\
\hline $\begin{array}{l}\text { ST-36 } \\
\text { Zusanli) }\end{array}$ & $\begin{array}{l}\text { On the posterior and lateral side of the knee joint, } \\
\text { mm below capitulum fibulae }\end{array}$ \\
\hline $\begin{array}{l}\text { PC-6 } \\
\text { (Neiguan) }\end{array}$ & $\begin{array}{l}\text { Three fingers width up from the wrist crease and } \\
\text { between the tendons of the flexor carpi radialis and } \\
\text { palmaris longus }\end{array}$ \\
\hline $\begin{array}{l}\text { DU-20 } \\
\text { (Baihui) }\end{array}$ & $\begin{array}{l}\text { On the top of the head at the intersection of middle } \\
\text { sagittal line and the line connecting two ears }\end{array}$ \\
\hline $\begin{array}{l}\text { GB-20 } \\
\text { (Fengchi) }\end{array}$ & $\begin{array}{l}\text { On the posterior neck, in the depression between } \\
\text { the upper portions of sternocleidomastoid and the } \\
\text { trapezius muscles }\end{array}$ \\
\hline $\begin{array}{l}\text { EX-8 } \\
\text { (Anmian I) }\end{array}$ & $\begin{array}{l}\text { Posterior to the ear, at the indentation next to the } \\
\text { mastoid process, between TB-I7 and GB-20 points }\end{array}$ \\
\hline
\end{tabular}

hygiene" recommendations discussed earlier. ${ }^{28}$ These educational points were repeated and discussed with the patients at every visit during follow-up to improve their adherence to the recommended points.

\section{Outcomes Assessment}

\section{Pittsburgh Sleep Quality Index (PSQI)}

The main study outcome was the quality of sleep measure by PSQI scale, which was filled out for both groups before and after the study period at the third week. PSQI is a questionnaire designed by Buysse et $\mathrm{al}^{29}$ as a selfreported multidimensional method for evaluating sleep quality in patients. This questionnaire consists of 19 questions investigating the subjects regarding seven domains of sleep quality: subjective sleep quality, sleep latency, sleep duration, habitual sleep efficiency, sleep disturbance, use of sleeping medications and daytime dysfunction over the last month. A higher PSQI score is associated with worse sleep quality in patients. Therefore, it is generally accepted that a patient with a PSQI score higher than 5 is suffering from a disturbed sleep pattern. ${ }^{30}$ In the current study, the Persian version of the PSQI questionnaire was used to collect the data. The study by Moghadam et $\mathrm{al}^{31}$ revealed that the Cronbach's alpha coefficient for the Persian version of PSQI was 0.77 . 


\section{Urine 6-Sulfatoxymelatonin Measurement}

Patients were instructed to collect first-spot morning urine samples at 6-8 AM. The urine sample was stored in a dark urine specimen container to prevent light exposure. Urine samples were centrifuged (at 2,500 rpm) for approximately 20 minutes and kept at $-20^{\circ} \mathrm{C}$ until analysis. Laboratory staff analyzing urine samples and reporting results were blinded to treatment groups. Urine 6-sulfatoxymelatonin was measured by commercial enzyme-linked immunosorbent assay (ELISA) kits (IBL International, Hamburg, Germany). It is well-elucidated that morning urine 6-sulfatoxymelatonin, the main metabolite of melatonin, is a useful measure of the total amount of melatonin produced overnight. ${ }^{32}$ The ratio of urine 6-sulfatoxymelatonin divided by urine creatinine $(\mathrm{Cr})$ level was expressed as $\mathrm{ng} / \mathrm{mg}$, in order to account for the differences in glomerular filtration rate among participants.

\section{Sample Size}

The strategy of sample size calculation was based on mean reduction of PSQI score $(-0.70 \pm 0.95)$ for the pilot sample, and the expectation of two more times reduction in acupuncture group (mean $=-1.4$ ). By considering $\alpha=0.05$, power $=80 \%$, and an approximately $20 \%$ dropout rate, 72 patients were examined and equally assigned to each group $(n=36)$.

\section{Statistical Analysis}

All statistical analyses were performed with the SPSS version 17.0 for Windows (SPSS Inc., Chicago, IL). Appropriately, data were presented as mean \pm standard deviation or frequency (percentage). Independent sample $t$-test or Mann Whitney $U$-tests were used for betweengroup comparison. Paired $t$-test or Wilcoxon signed-rank tests were used for within-group comparisons. The standardized mean differences (SMDs) with $95 \%$ confidence intervals (CIs) was used to estimate the effect size of intervention. An effect size of $\mathrm{d}=0.2$ is considered a small effect size, $\mathrm{d}=0.5$ represents a medium effect size, and 0.8 or above a large effect size. A $P$-value less than 0.05 was considered statistically significant.

\section{Results}

Seventy-two pregnant women with insomnia were randomly assigned to the intervention $(n=36)$ and control $(n=36)$ groups. As shown in Figure 1, 55 of 72 study participants completed the trial.

According to Table 2, the mean age of participants was $31.7 \pm 5.7$ years for the intervention group and $30.2 \pm 3.4$ years for the control group $(P=0.267)$. Gestational age, documented by obstetrical sonography and reported by weeks, at the beginning of the study was similar across the study groups $(P=0.157)$. There were no significant differences between the study groups in terms of body mass index (BMI), educational level, occupation status, and gravidity $(P>0.05)$.

Baseline PSQI scores for the intervention and control groups were $12.7 \pm 1.8$ and $12.2 \pm 1.8$, respectively, which showed no significant difference $(P=0.169)$. Regarding between-group analysis, the study subjects obtained significantly lower PSQI scores in the intervention group (6.0 $\pm 1.1)$ compared to the control group $(12.1 \pm 2.0)$ at the end of the intervention $(P<0.001)$. This lower PSQI score in the intervention group indicates a better sleep quality for those receiving real acupuncture. These results are summarized in Table 3.

Data regarding the acupuncture and urinary level of 6-Sulfatoxymelatonin is listed in Table 4. At baseline, urinary 6-Sulfatoxymelatonin level was $19.8 \pm 5.0 \mathrm{ng} / \mathrm{mg}$ in the intervention group and $20.7 \pm 4.9 \mathrm{ng} / \mathrm{mg}$ in the control group, which showed no significant difference $(P=0.496)$. However, between-group analysis revealed a significant difference between the acupuncture and control groups after the intervention in the 6-Sulfatoxymelatonin level (27.4 \pm 6.5 $\mathrm{ng} / \mathrm{mg}$ vs $22.8 \pm 7.6 \mathrm{ng} / \mathrm{mg}, P=0.020$ ).

The effect size of intervention on sleep quality (PSQI) and 6-Sulfatoxymelatonin level was reported to be 3.7 $(\mathrm{CI}=2.8-4.6)$ and $0.6 \quad(\mathrm{CI}=0.1-1.2)$, which revealed a large $(>0.8)$ and medium $(>0.5)$ effect size, respectively.

No serious acupuncture-related adverse events (AEs) were recorded during the study. The only reported AE was needling pain. No participant in either group was reported to take hypnotic medication for insomnia during the studyperiod. Neonatal AEs were also evaluated. One newborn was admitted to the neonatal intensive care unit (NICU) from the intervention group due to transient tachypnea and two newborns from the control group due to neonatal sepsis and hyperbilirubinemia. Thus, these AEs seem not to be attributable to the use of acupuncture.

\section{Discussion}

There is a growing interest for using acupuncture in treatment of insomnia in the general population. ${ }^{13}$ However, data on its effectiveness in pregnant women with insomnia is sparse. The term "pregnancy-related insomnia" differs from insomnia during other stages of life, due to different pathological or physiological conditions leading to 


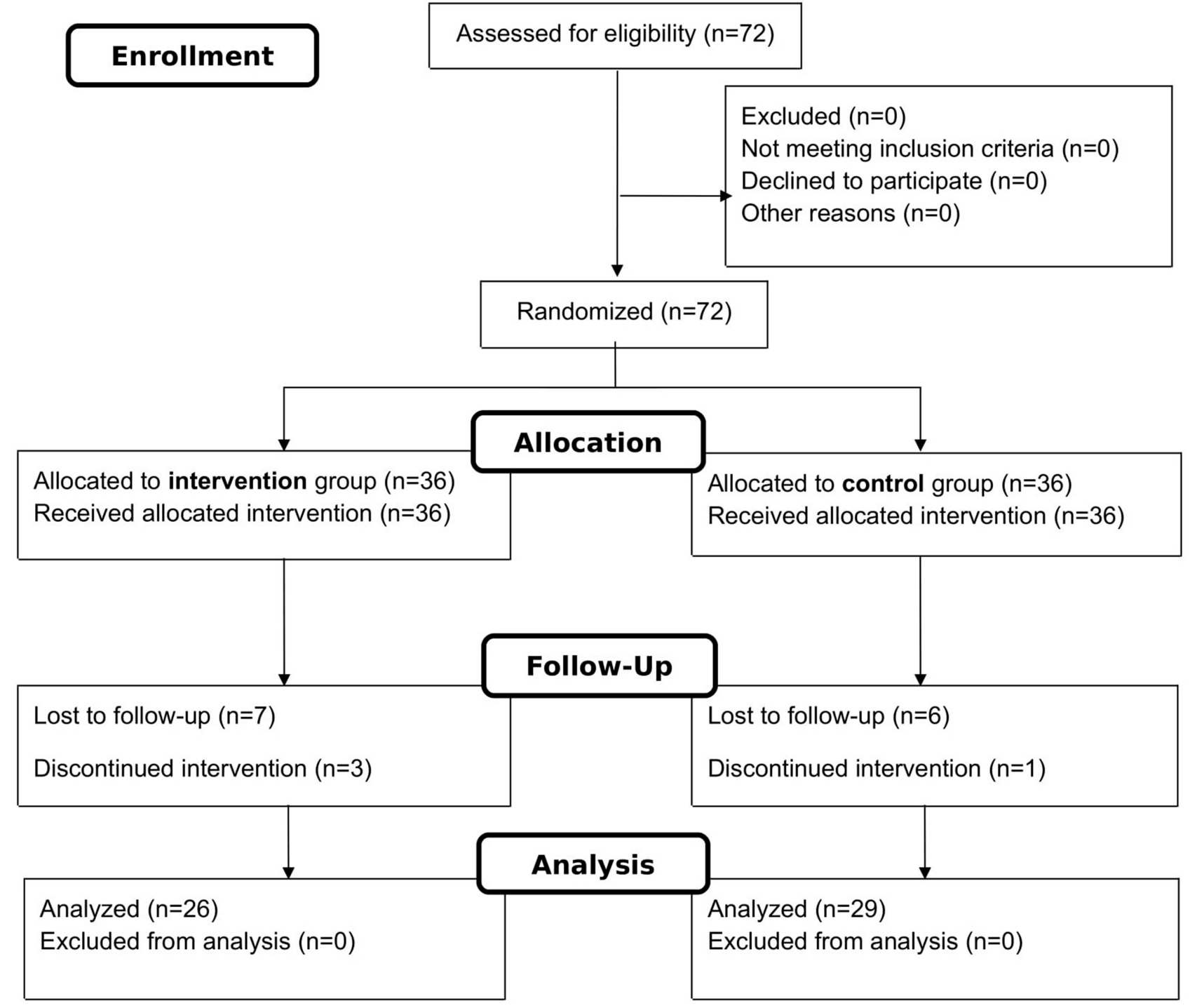

Figure I CONSORT flow diagram for the design of this clinical trial.

a disturbance in sleep pattern of pregnant women. ${ }^{33}$ To the best of our knowledge, this is the first randomized placebo-controlled trial evaluating acupuncture efficacy and safety for pregnancy-related insomnia through both subjective and laboratory (melatonin) measurements.

In our study, the acupuncture treatment resulted in a significant improvement of 6.7 points in total PSQI score among pregnant women. This finding is consistent with the results of a previous study that found acupuncture for 8-12 sessions improved sleep quality in pregnant women. ${ }^{14}$ However, utilization of a validated measurement tool (eg, validated questionnaire) as well as a placebo (sham)-controlled group was not mentioned.

We observed an increase in endogenous melatonin secretion (as measured in morning spot urine) after acupuncture treatment, which was followed by improvement in sleep quality in participants, assessed by PSQI, in the current study. However, it is too early to claim a causal relationship between increasing melatonin after acupuncture and improvement in sleep quality at this stage. Spence et $\mathrm{al}^{17}$ obtained similar results, showing a significant increase in urinary melatonin excretion after performing acupuncture on 18 adult subjects with anxiety disorder suffering from insomnia.

Acupuncture efficacy could be attributable to its regulatory effect on various neuroendocrinological pathways. It has been shown that stimulation of certain acupoints resulted in a change in a wide range of neuroendocrinological mediators, such as norepinephrine, melatonin, gamma-aminobutyric acid, and $\beta$-endorphin. ${ }^{34}$ Accordingly, in an animal study, manual 
Table 2 Demographic Characteristics of Study Groups

\begin{tabular}{|l|l|l|l|}
\hline Characteristics & $\begin{array}{l}\text { Intervention } \\
(\mathbf{n = 2 6 )}\end{array}$ & $\begin{array}{l}\text { Control } \\
(\mathbf{n = 2 9 )}\end{array}$ & P-value $^{\mathbf{a}}$ \\
\hline $\begin{array}{l}\text { Maternal age, years } \\
\text { Bostational age, weeks } \\
\text { (BMI), kg/m }\end{array}$ & $31.7 \pm 5.7$ & $30.2 \pm 3.4$ & 0.267 \\
\hline $\begin{array}{l}\text { Education } \\
\text { - Diploma or under } \\
\text { diploma }\end{array}$ & $14(53.8 \%)$ & $23.2 \pm 7.2$ & 0.157 \\
- University degree & $12(46.2 \%)$ & $12(41.4 \%)$ & 0.355 \\
\hline $\begin{array}{l}\text { Gravidity } \\
\text { I }\end{array}$ & & $17(58.6 \%)$ & \\
\hline 2 & $6(23 \%)$ & $9(31 \%)$ & 0.656 \\
$\bullet \geq 3$ & $12(46.2 \%)$ & $10(34.5 \%)$ & \\
\hline $\begin{array}{l}\text { Occupation } \\
\text { - Housewife }\end{array}$ & $8(30.8 \%)$ & $10(34.5 \%)$ & \\
\hline Employed & $24(92.3 \%)$ & $23(79.3 \%)$ & 0.256 \\
\hline
\end{tabular}

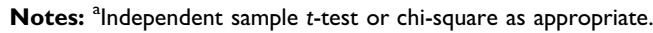
Abbreviation: BMI, body mass index.

Table 3 Comparison of Total PSQI Scores between the Two Groups before and after Intervention

\begin{tabular}{|c|c|c|c|}
\hline \multirow[t]{2}{*}{$\begin{array}{l}\text { Total PSQI } \\
\text { Score }\end{array}$} & $\begin{array}{l}\text { Intervention } \\
(n=26)\end{array}$ & $\begin{array}{l}\text { Control } \\
(n=29)\end{array}$ & $P$-value ${ }^{a}$ \\
\hline & Mean $\pm S D$ & Mean士SD & \\
\hline Before treatment & $12.7 \pm 1.8$ & $12.2 \pm 1.8$ & 0.169 \\
\hline After treatment & $6.0 \pm 1.1$ & $12.1 \pm 2.0$ & $<0.001$ \\
\hline$P$-value ${ }^{b}$ & $<0.001$ & 0.326 & \\
\hline
\end{tabular}

Notes: andependent sample $t$-test. ${ }^{\mathrm{b}}$ Paired sample $t$-test. Abbreviation: PSQI, Pittsburgh Sleep Quality Index.

Table 4 Comparison of Urinary 6-Sulfatoxymelatonin/Creatinine Ratio between the Two Groups before and after Intervention

\begin{tabular}{|c|c|c|c|}
\hline \multirow{2}{*}{$\begin{array}{l}\text { Urine } \\
\text { 6-Sulfatoxymelatonin } \\
\text { (ng/mg Cr) }\end{array}$} & $\begin{array}{l}\text { Intervention } \\
(n=26)\end{array}$ & $\begin{array}{l}\text { Control } \\
(n=29)\end{array}$ & \multirow[t]{2}{*}{$P$-value ${ }^{a}$} \\
\hline & Mean $\pm S D$ & Mean $\pm S D$ & \\
\hline Before treatment & $19.8 \pm 5.0$ & $20.7 \pm 4.9$ & 0.496 \\
\hline After treatment & $27.4 \pm 6.5$ & $22.8 \pm 7.6$ & 0.020 \\
\hline$P$-value ${ }^{b}$ & $<0.001$ & 0.147 & \\
\hline
\end{tabular}

Notes: ${ }^{a}$ Independent sample $t$-test. ${ }^{b}$ Paired sample $t$-test.

Abbreviations: ng, nanograms; mg, milligrams; $\mathrm{Cr}$, creatinine.

stimulation of Baihui, Shenman, and Sanyinjiao acupoints was shown to improve the sleep duration in insomniac rats. This promising effect of acupuncture was attributable to its effect on increasing the expression level of melatonin protein, as well as melatonin-1 and melatonin-2 mRNAs in rat models. ${ }^{18}$
Melatonin crosses the placenta freely and plays an important role in fetal development. Maternal serum melatonin is shown to regulate fetal rapid eye movement (REM) and non-REM sleep cycle during the third trimester of pregnancy, ${ }^{35}$ which is directly associated with fetal brain neuronal growth and activation during this period of time. ${ }^{36}$ Therefore, increased melatonin secretion following acupuncture may not only improve maternal sleep quality, but also have the potential to positively affect fetal brain development.

A previous study has shown that women have an increased desire and awareness to experience acupuncture, as an alternative treatment, in both antenatal and postnatal periods, and those who received acupuncture treatment during pregnancy reported positive feedback regarding the effectiveness of this therapy. ${ }^{37}$ Acupuncture is considered generally safe during pregnancy. ${ }^{27}$ One proof for this claim is that several studies conducted for treatment of low back and pelvic pain, tension type headache, dyspepsia, nausea and vomiting, anxiety and depression during pregnancy have shown no serious adverse effects related to the acupuncture. $^{38,42}$

In addition to modern medicine, Traditional Chinese Medicine (TCM) theories have been used to explain that acupuncture treatment may be regulating yin and yang to reinforce improving sleep quality. ${ }^{13}$ The diagnosis made according to TCM theories has been suggested to be related to acupuncture response in patients. For instance, particular features of TCM diagnoses were predictors of treatment response to acupuncture used for hot flashes in patients suffering from cancer. ${ }^{43}$ Therefore, it may be logical to select patients for acupuncture based on an individualized process, in order to optimize the response obtained from treatment.

This study has some limitations: 1) Study subjects were not followed-up for a long-period to see whether or not the efficacy of treatment was persisting for longer periods of time. 2) Acupuncturist could not be blinded to the assigned groups due to the nature of intervention. 3) The relatively small number of participants analyzed at the end of study $(n=55)$, which limits the generalizability of the results. 4) The PSQI questionnaire is a subjective tool, and may not accurately reflect sleep quality, so further trials using objective measurement tools are recommended.

\section{Conclusion}

Results of the current trial suggest that acupuncture treatment significantly improves the sleep quality in pregnant women, 
possibly through increasing melatonin secretion. Further randomized clinical trials with larger sample sizes and longer follow-up periods are needed to confirm these results.

\section{Data Sharing Statement}

The data that support the findings of this study are available from the corresponding author upon reasonable request. Also, the individual deidentified participant data is available after contacting the corresponding author via email. The data will be available immediately following publication without an end date.

\section{Acknowledgments}

This article was extracted from the thesis written by Saeede Foroughinia for a degree of Obstetrics and Gynecology, and was financially supported by the Shiraz University of Medical Sciences.

\section{Author Contributions}

All authors contributed to data analysis, drafting or revising the article, gave final approval of the version to be published, and agree to be accountable for all aspects of the work.

\section{Funding}

The project was financed by Vice Chancellor for Research of the Shiraz University of Medical Science, Shiraz, Iran. The funding organization played no role in the study design, data collection, analysis, and interpretation of data.

\section{Disclosure}

The authors declare that they have no conflicts of interest.

\section{References}

1. Sedov ID, Cameron EE, Madigan S, Tomfohr-Madsen LM. Sleep quality during pregnancy: a meta-analysis. Sleep Med Rev. 2018;38:168-176. doi:10.1016/j.smrv.2017.06.005

2. Garbazza C, Hackethal S, Riccardi S, et al. Polysomnographic features of pregnancy: a systematic review. Sleep Med Rev. 2020;50:101249. doi:10.1016/j.smrv.2019.101249

3. Habr F, Raker C, Lin CL, Zouein E, Bourjeily G. Predictors of gastroesophageal reflux symptoms in pregnant women screened for sleep disordered breathing: a secondary analysis. Clin Res Hepatol Gastroenterol. 2013;37(1):93-99. doi:10.1016/j.clinre.2012.03.036

4. Chang JJ, Pien GW, Duntley SP, Macones GA. Sleep deprivation during pregnancy and maternal and fetal outcomes: is there a relationship? Sleep Med Rev. 2010;14(2):107-114. doi:10.1016/j.smrv.2009.05.001

5. Warland J, Dorrian J, Morrison JL, O'Brien LM. Maternal sleep during pregnancy and poor fetal outcomes: a scoping review of the literature with meta-analysis. Sleep Med Rev. 2018;41:197-219. doi:10.1016/j.smrv.2018.03.004
6. Zhao Q, Xie X, Fan Y, et al. Phenotypic dysregulation of microglial activation in young offspring rats with maternal sleep deprivation-induced cognitive impairment. Sci Rep. 2015;5:9513. doi:10.1038/srep09513

7. Pardo GV, Goularte JF, Hoefel AL, et al. Effects of sleep restriction during pregnancy on the mother and fetuses in rats. Physiol Behav. 2016;155:66-76. doi:10.1016/j.physbeh.2015.11.037

8. Okun ML, Ebert R, Saini B. A review of sleep-promoting medications used in pregnancy. Am J Obstet Gynecol. 2015;212(4):428-441.

9. Juric S, Newport DJ, Ritchie JC, Galanti M, Stowe ZN. Zolpidem (Ambien) in pregnancy: placental passage and outcome. Arch Womens Ment Health. 2009;12(6):441-446.

10. Wikner BN, Stiller CO, Bergman U, Asker C, Kallen B. Use of benzodiazepines and benzodiazepine receptor agonists during pregnancy: neonatal outcome and congenital malformations. Pharmacoepidemiol Drug Saf. 2007;16(11):1203-1210. doi:10.1002/pds.1457

11. Reichner CA. Insomnia and sleep deficiency in pregnancy. Obstet Med. 2015;8(4):168-171. doi:10.1177/1753495X15600572

12. Briggs GG, Freeman RK, Yaffe SJ. Drugs in Pregnancy and Lactation: A Reference Guide to Fetal and Neonatal Risk. Lippincott Williams \& Wilkins; 2012.

13. Cao H, Pan X, Li H, Liu J. Acupuncture for treatment of insomnia: a systematic review of randomized controlled trials. $J$ Altern Complement Med. 2009;15(11):1171-1186. doi:10.1089/acm.2009.0041

14. da Silva JB, Nakamura MU, Cordeiro JA, Kulay LJ. Acupuncture for insomnia in pregnancy-a prospective, quasi-randomised, controlled study. Acupunct Med. 2005;23(2):47-51. doi:10.1136/aim.23.2.47

15. Fu C, Zhao N, Liu Z, et al. Acupuncture improves peri-menopausal insomnia: a randomized controlled trial. Sleep. 2017;40(11). doi:10.1093/sleep/zsx153.

16. Wang H, Yang G, Wang S, Zheng X, Zhang W, Li Y. The most commonly treated acupuncture indications in the United States: a cross-sectional study. Am J Chin Med. 2018;46:1-33.

17. Spence DW, Kayumov L, Chen A, et al. Acupuncture increases nocturnal melatonin secretion and reduces insomnia and anxiety: a preliminary report. J Neuropsychiatry Clin Neurosci. 2004;16 (1):19-28. doi:10.1176/jnp.16.1.19

18. Zheng XN, Wu XF, Guo X, et al. [Manual acupuncture stimulation of paired acupoints can relieve sleep disorder possibly by upregulating pineal melatonin protein and its receptor mRNA levels in the suprachiasmatic nucleus in insomnia rats]. Acupunt Res. 2018;43 (6):360-364. doi:10.13702/j.1000-0607.170409

19. Suzuki S, Dennerstein L, Greenwood KM, Armstrong SM, Sano T, Satohisa E. Melatonin and hormonal changes in disturbed sleep during late pregnancy. $J$ Pineal Res. 1993;15(4):191-198. doi:10.1111/j.1600-079X.1993.tb00904.x

20. Schulz KF, Altman DG, Moher D. CONSORT 2010 statement: updated guidelines for reporting parallel group randomised trials. BMC Med. 2010;8(1):18. doi:10.1186/1741-7015-8-18

21. MacPherson H, Altman DG, Hammerschlag R, et al. Revised standards for reporting interventions in clinical trials of acupuncture (STRICTA): extending the CONSORT statement. J Evid Based Med. 2010;3(3):140-155. doi:10.1111/j.1756-5391.2010.01086.x

22. Sateia MJ. International classification of sleep disorders-third edition: highlights and modifications. Chest. 2014;146(5):1387-1394. doi:10.1378/chest.14-0970

23. Grundy A, Sanchez M, Richardson H, et al. Light intensity exposure, sleep duration, physical activity, and biomarkers of melatonin among rotating shift nurses. Chronobiol Int. 2009;26(7):1443-1461. doi: $10.3109 / 07420520903399987$

24. Park J, White A, Stevinson C, Ernst E, James M. Validating a new non-penetrating sham acupuncture device: two randomised controlled trials. Acupunct Med. 2002;20(4):168-174. doi:10.1136/aim.20.4.168

25. Streitberger K, Kleinhenz J. Introducing a placebo needle into acupuncture research. Lancet. 1998;352(9125):364-365. doi:10.1016/ S0140-6736(97)10471-8 
26. Yin X, Gou M, Xu J, et al. Efficacy and safety of acupuncture treatment on primary insomnia: a randomized controlled trial. Sleep Med. 2017;37:193-200. doi:10.1016/j.sleep.2017.02.012

27. Park J, Sohn Y, White AR, Lee H. The safety of acupuncture during pregnancy: a systematic review. Acupunct Med. 2014;32(3):257-266. doi:10.1136/acupmed-2013-010480

28. Irish LA, Kline CE, Gunn HE, Buysse DJ, Hall MH. The role of sleep hygiene in promoting public health: a review of empirical evidence. Sleep Med Rev. 2015;22:23-36. doi:10.1016/j.smrv.2014.10.001

29. Buysse DJ, Reynolds CF 3rd, Monk TH, Berman SR, Kupfer DJ. The pittsburgh sleep quality index: a new instrument for psychiatric practice and research. Psychiatry Res. 1989;28(2):193-213. doi:10.1016/0165-1781(89)90047-4

30. Backhaus J, Junghanns K, Broocks A, Riemann D, Hohagen F. Testretest reliability and validity of the pittsburgh sleep quality index in primary insomnia. J Psychosom Res. 2002;53(3):737-740. doi:10.1016/S0022-3999(02)00330-6

31. Farrahi Moghaddam J, Nakhaee N, Sheibani V, Garrusi B, Amirkafi A. Reliability and validity of the Persian version of the Pittsburgh Sleep Quality Index (PSQI-P). Sleep Breath. 2012;16 (1):79-82. doi:10.1007/s11325-010-0478-5

32. Bojkowski C, Aldhous M, English J, et al. Suppression of nocturnal plasma melatonin and 6-sulphatoxymelatonin by bright and dim light in man. Horm Metab Res. 1987;19(09):437-440. doi:10.1055/s-20071011846

33. Pien GW, Schwab RJ. Sleep disorders during pregnancy. Sleep. 2004;27(7):1405-1417. doi:10.1093/sleep/27.7.1405

34. Zhao K. Acupuncture for the treatment of insomnia. In: Int Rev Neurobiol. 2013;111:217-234doi:10.1016/b978-0-12-411545-3.00011-0.

35. Tamura H, Nakamura Y, Terron MP, et al. Melatonin and pregnancy in the human. Reprod Toxicol. 2008;25(3):291-303. doi:10.1016/j. reprotox.2008.03.005
36. Morrissey MJ, Duntley SP, Anch AM, Nonneman R. Active sleep and its role in the prevention of apoptosis in the developing brain. Med Hypotheses. 2004;62(6):876-879. doi:10.1016/j.mehy.2004.01.014

37. Williams H, Sweet L, Graham K. Acupuncture during pregnancy and the perinatal period: women's attitudes, beliefs and practices. Women Birth. 2019

38. Ternov NK, Grennert L, Aberg A, Algotsson L, Akeson J. Acupuncture for lower back and pelvic pain in late pregnancy: a retrospective report on 167 consecutive cases. Pain Med. 2001;2 (3):204-207. doi:10.1046/j.1526-4637.2001.01031.x

39. Smith C, Crowther C, Beilby J. Pregnancy outcome following women's participation in a randomised controlled trial of acupuncture to treat nausea and vomiting in early pregnancy. Complement Ther Med. 2002;10(2):78-83. doi:10.1054/ctim.2002.0523

40. Smith CA, Shewamene Z, Galbally M, Schmied V, Dahlen H. The effect of complementary medicines and therapies on maternal anxiety and depression in pregnancy: a systematic review and meta-analysis. J Affect Disord. 2019;245:428-439. doi:10.1016/j.jad.2018.11.054

41. da Silva JB, Nakamura MU, Cordeiro JA, Kulay L Jr, Saidah R. Acupuncture for dyspepsia in pregnancy: a prospective, randomised, controlled study. Acupunct Med. 2009;27(2):50-53. doi:10.1136/ aim.2009.000497

42. JBGd S, Nakamura MU, Cordeiro JA, Kulay L. Acupuncture for tension-type headache in pregnancy: a prospective, randomized, controlled study. Eur J Integr Med. 2012;4(4):e366-e370. doi:10.1016/j. eujim.2012.04.002

43. Liu W, Qdaisat A, Lopez G, et al. Acupuncture for hot flashes in cancer patients: clinical characteristics and traditional chinese medicine diagnosis as predictors of treatment response. Integr Cancer Ther. 2019;18:1534735419848494. doi:10.1177/1534735419848494
Nature and Science of Sleep

\section{Publish your work in this journal}

Nature and Science of Sleep is an international, peer-reviewed, open access journal covering all aspects of sleep science and sleep medicine, including the neurophysiology and functions of sleep, the genetics of sleep, sleep and society, biological rhythms, dreaming, sleep disorders and therapy, and strategies to optimize healthy sleep.

\section{Dovepress}

The manuscript management system is completely online and includes a very quick and fair peer-review system, which is all easy to use. Visit http://www.dovepress.com/testimonials.php to read real quotes from published authors. 